\title{
Ordered layered organic-inorganic of 4-chlorophenoxyacetate-zinc layered hydroxide nanohybrid
}

\begin{abstract}
Ordered layered organic-inorganic nanostructure composed of zinc layered hydroxide-4chlorophenoxy acetate (ZLH-4CPA) was prepared by reaction of an organic anion, 4chlorophenoxy acetate (4CPA) with $\mathrm{ZnO}$ under aqueous environment. The concentration of 4CPA was found to be a controlling factor in determining the formation of phase pure, well ordered nanolayered hybrid material. At lower concentration of 4CPA (0.05 M), a mixed phase was observed in which $\mathrm{ZnO}$ co-existed with the nanohybrid. At $0.01 \mathrm{M}$, a pure phase is obtained with high crystallinity but a well ordered nanolayered structure is lacking. A pure phase, well ordered nanolayered hybrid can be clearly observed at $0.2 \mathrm{M} 4 \mathrm{CPA}$. ZnO shows well defined grain structure of various sizes at nanometer scale range. Direct reaction between $\mathrm{ZnO}$ and 4CPA under aqueous environment resulted in the formation of 4CPA-ZLH nanohybrid with flake-like fibrous structure. On heating at $500^{\circ}$ [Cl for $5 \mathrm{~h}$ under atmospheric condition, the nanohybrid was transformed back to well defined grain structure, as previously observed for the starting materials, $\mathrm{ZnO}$. This shows that the nanohybrid has ñmemory effectò property. Well ordered nanolayered hybrid with up to 5 harmonics, from which the average basal spacing of $19.03 \AA$ of the material was deduced, showing long range order of the layer packing.
\end{abstract}

Keyword: 4-chlorophenoxyacetate; Memory effect property; Nanohybrid; Zinc layered hydroxide 